\title{
An Improved Endpoint Detection Algorithm using Bit Wise Approach for Isolated, Spoken Paired and Hindi Hybrid Paired Words
}

\author{
Taabish Gulzar \\ M.Tech. Student \\ Department of Electronics \& \\ Communication Engineering \\ D.I.T., Dehardun, India
}

\author{
Anand Singh \\ Assistant Professor \\ Department of Electronics \& \\ Communication Engineering \\ D.I.T., Dehradun, India
}

\author{
Sandip Vijay \\ Ph. D, Professor \& HOD \\ Department of Electronics \& \\ Communication Engineering \\ D.I.T., Dehradun, India
}

\begin{abstract}
Silence removal and endpoint detection using preset threshold values have already been used for locating the endpoints of an utterance. This paper presents a survey of bit by bit basis method for detecting the accurate endpoints of Isolated, Spoken Paired and Spoken Hindi Hybrid Paired words. Various parameters such as Number of samples, Time duration, Root Mean Square value and Mean Power (Intensity) in air are analyzed. The experiment results show that the proposed algorithm reduces the computational complexity.
\end{abstract}

\section{General Terms}

Pre-Processing, Recognition rate, Bit-Wise, Zero crossing rate, Speech Recognition.

\section{Keywords}

Bit wise analysis, endpoint detection, Paired words, Spoken Hindi Hybrid word.

\section{INTRODUCTION}

One of the most important ways of communication among humans is language and primary medium used for this communication is speech. Speech recognition via machines finds its applications in almost all sectors. Researchers are on their way for developing the systems that can provide better response to the users. The complicated assignment of speech processing has been divided in three relatively easier categories, (a) speech recognition: that allows the machines to understand the words, sentences, phrases spoken by humans, (b) Natural language processing: that allows the machines to understand the desire of humans, (c) speech synthesis: that allows the machines to respond back to users $[1,2]$. Speech produced by same speaker different times, differ in the parameters containing within it such as energy, pitch, etc. For developing a robust speech system it is necessary to characterize the emotions present within a speech signal [3]. A unique category of words known as Spoken words are of different types such as short words, moderate words and long words [4].Spoken paired words have an edge over short and long words, as for as misrecognition rate, processing time, memory allocation is concerned [5]. Paired words overcome these problems as they contain moderate word length that requires less computation time and memory allocation. A gap of several milliseconds is always found in Paired words that play a vital role in recognition process, which depends on many factors, e.g. (length of vocal tract, air pressure through lungs, emotions of speaker, etc [6, 7]. In case of Hindi connected words only Hindi language is used for recognition purpose. However, to strengthen the speech code (gap between two words) two different languages are used. Hence a new category of words called Spoken Hindi Hybrid Paired words came in existence [8]. Hindi Hybrid words refer to the words in which one word is necessary from Hindi and other may be from any English, Urdu, Farsi, Sanskrit etc.

Pre-Processing of speech is essential in the applications where silence or background noise is entirely undesirable. The detection of the occurrence of speech segment embedded in different categories of non speech events and background noise is called endpoint detection, voice activity detection (VAD) or Silence removal. Silence removal techniques have been studied for several years. VAD finds its very first application in telephone transmission and switching system developed in Bell Labs, for the assignment of communication channel, where the channels idle time is detected by the presence of users speech and consequently an unused channel is assigned when speech is detected, providing more customer services [9]. The falsely detected endpoints of an utterances results in at least two negative effects [10]:

1. Recognition errors are introduced due to the inaccurate boundaries of the speech.

2. Increases the computation if inaccurate boundaries are detected.

Two broadly accepted methods namely Short Time Energy and Zero Crossing Rate (ZCR) have been used since a long time for removing the background noise from speech [11, 12]. Unfortunately these algorithms have some problems regarding setting the thresholds as ad hoc basis. This value of threshold is often assumed to be fixed or computed in voice-inactive (silence) intervals of a speech signal [13].

A newer promising approach involves the use of Bit-Wise analysis to locate the accurate endpoints where the concept of setting the threshold as ad hoc basis is avoided. The proposed method reduces the computational complexity and at the same time it does not make use of ZCR, which sometimes is not feasible. Another advantage of using this method is that there is no need for the calculation of background noise. Hence it is efficient for the environment, where there is strong noise at the beginning or ending of the speech or when the speech artifacts such as breath, mouth and lip clicks are likely to happen which in turn locate the false boundaries.

The rest of the paper is organized as follows: In Section II, we will introduce the theoretical background. Section III briefly describes the Bit-wise algorithm for evaluating the endpoints 
of the utterances. Section IV presents the database preparation and experimental results. Finally, we will summary our findings in section IV.

\section{THEORETICAL BACKGROUND}

Speech signal is a slowly time varying signal, when seen over a relatively short interval of time, and its characteristics are quite stationary [14]. In order to meet the requirements that include computational accuracy, complexity, response time etc different applications make use of different algorithms. These applications include those which are based on energy threshold, pitch detection, ZCR [15, 16, 17, 18].The end points of speech are usually obscured by speaker generated artifacts such as clicks or by dial tone. Long-distance telephone transmission channels may also introduce these types of artifacts and some background noise [19]. Conventional short-time or spectral energy or ZCR based endpoint detection algorithms are usually susceptible to speech artifacts such as breath, mouth and lip clicks and break down easily in the presence of noise. These classical energy threshold methods i.e. energy and ZCR methods, the threshold value need to be recalculated at each and every silence (voiceinactive) segment [20]. And in case, the noise is nonstationary, these methods fail to track the exact value of thresholds, resulting in falsely detected endpoints. In some of the applications which may include speaker verification, name dialing, speech control etc, where the speech (voice-active) part of the signal is sometimes less (e.g. less than $2 \mathrm{~s}$ ) and the recognition process can be done within $1 \mathrm{~s}$ or even less, that are usually provided by embedded systems, such as wireless phones or portable devices; or in multi-user systems, such as speaker verification system for several users, usually a low computational complexity for low cost or for faster response of the system is required [21]. One solution for the abovementioned cases is to make use of an accurate endpoint detection algorithm to remove all silence (voice-inactive) part. Intrusion of the proposed Bit-wise method uniquely defines the threshold first instance.

\section{PROPOSED ALGORITHM}

In this section, an algorithm for endpoint detection that makes use of bit by bit comparison of the signal is presented and meets the following requirements: accurate detection of endpoints, low computational complexity, fast response time and simple steps of implementations.

Step 1: Removing zeroes from the signal:

The adjacent bits of the speech signal are checked and zeroes i.e. the silence (voice-inactive) intervals are removed.

Step 2: Calculation of threshold:

The signal is then transposed. If $\mu$ and $\sigma$ are the values of mean and standard deviation respectively then mathematically,

$$
\begin{aligned}
& \sigma=\sqrt{1 / N \sum_{i=1}^{N}\left(x_{i}-\mu\right)^{2}} \\
& \mu=\sum_{i=1}^{N} x_{i}
\end{aligned}
$$

Where, $\mathrm{x}_{i}=$ observed values of the sample item,
$\mathrm{N}=$ size of the sample.

Step 3: Finding the start point of signal:

Once a threshold has been estimated, anything above this threshold is considered to be speech and anything below this optimum point is either noise or silence.

Signal $=\left\{\begin{array}{l}\text { speech, if signal (i) } \geq \text { threshold, } \mathrm{i}=1,2, \ldots \mathrm{n} \\ \text { silence, otherwise }\end{array}\right.$

If the difference between the successive bits of the signal is greater than the threshold value calculated, it is the start point of the signal and the signal is stored in that case.

Step 4: Finding the endpoint of the signal:

The threshold calculated in Step 2 is taken for estimating the endpoint of the signal. Now, if the difference between any of the bit and its preceding one is greater than the threshold calculated, this point is treated as the endpoint of the signal, and is stored.

Step 5: The resulting signal is the accurately endpoint detected signal.

\subsection{Set Up}

Step (1):- Initialize required variables

Step (2):- Read file, find length of the signal, Remove one channel.

Algorithm for endpoint detection:-

Step (3):- Remove Zeroes from the signal.

for $i \leftarrow 1$ to $r$

if $\leftarrow y(i)$ not 0

$y y(k) \leftarrow y(i)$

$k \leftarrow k+1$

end if

end for

Step (4):- Transpose signal to find end point.

Step (5):- Calculate threshold (Thold)

for $i \leftarrow 1$ to $r$

$m=m+(y y(i)-m e a n(y y))^{\wedge} 2 ;$

end for

$$
\text { Thold }=\operatorname{sqrt}(1 / r * m) \text {; }
$$

Step (6):- Find the start point.

for $i \leftarrow 1$ to $r$

if $y y(i)-y y(i+1)$ greater than Thold

break;

end if 
end for

Step (7):- Store remaining signal.

$y y y \leftarrow y y(1$ to $r, 1)$

Step (8):- Calculate threshold as calculated in Step (5).

Step (9):-Find the endpoint.

for $i \leftarrow r$ to 2 step -1

if yyy $(i-1)-y y y(i)$ greater than Thold

break;

end if

end for

Step (10):- Store the remaining signal as EPDsignal.

EPDsignal $\leftarrow$ yyy $(1$ to $r)$

Step (11):- The resultant signal is the endpoint detected signal

\section{DATABASE DEVELOPMENT AND EXPERIMENTAL RESULTS.}

Thirty different words are used for database, out of which ten words are Isolated, ten Spoken paired, and the remaining ten spoken Hindi hybrid words. Five female and five male speakers are taken for recording session, thus making a total of three hundred utterances. Stereo headset H 250 with frequency response of $20 \mathrm{~Hz}-20 \mathrm{KHz}$ was used. A sampling rate of $16000 \mathrm{~Hz}$ was used for all data. For some of the recordings there is some background or some lip and mouth clicks or breath noise.

The Pre-processing, endpoint detection technique was implemented and verified in MATLAB and PRAAT software's respectively. To evaluate performance, we visually compare the locations of the beginning and ending points using Bit wise and preset threshold approach. The detected endpoint signal is verified in PRAAT software for analyzing various parameters i.e. Number of samples (NOS), Time duration (TD) in seconds, Root mean square (RMS) in Pascal and Mean power intensity in air (MI) in dB. Figure 1 to figure 18 compares the waveforms of Isolated word (TEEN), Spoken Paired word (HUM-TUM) and Hybrid word (KHAS-AAMI) in their original form and endpoint detected form using Bit wise and preset threshold approaches for both female and male speakers respectively. Table 1 to Table 6 shows the average value of various parameters i.e. NOS, TD, RMS, MI for Isolated, Paired and Hybrid words for both female and male speakers respectively. Table 7 to Table 12 gives the increment and decrement in the abovementioned parameters for both female and male speakers respectively using Bit wise approach. Proposed algorithm performs well than conventional threshold method.

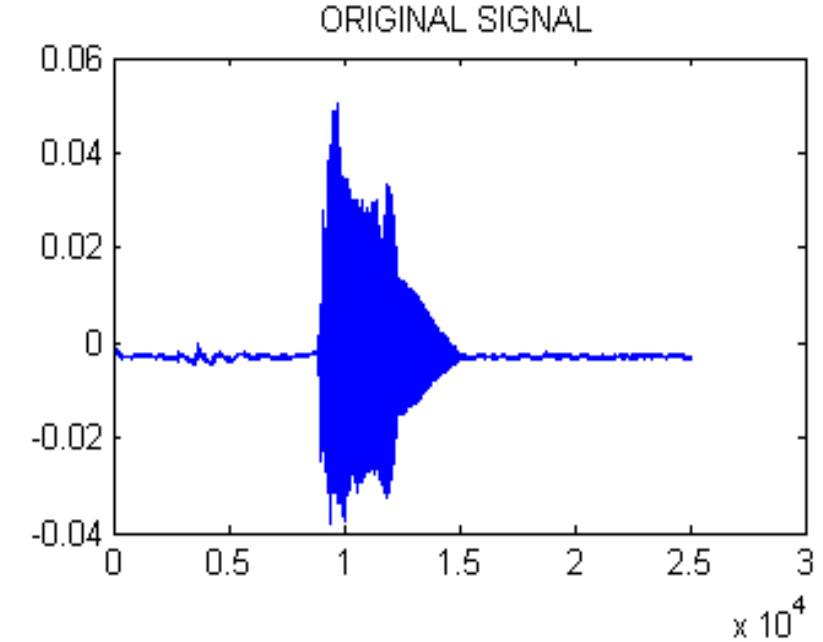

Figure 1: Waveform of isolated word (TEEN) by female speaker in the original form.

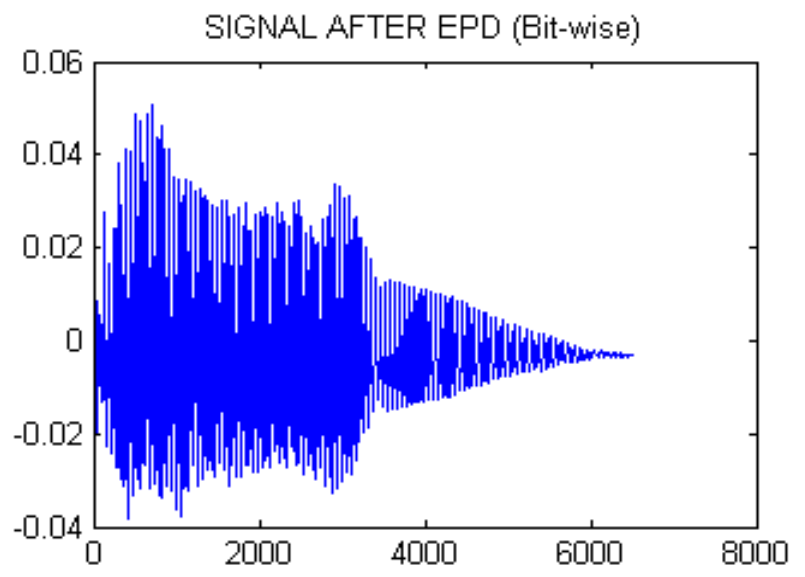

Figure 2: Waveform of isolated word (TEEN) by female speaker after endpoint detection (Bit wise).

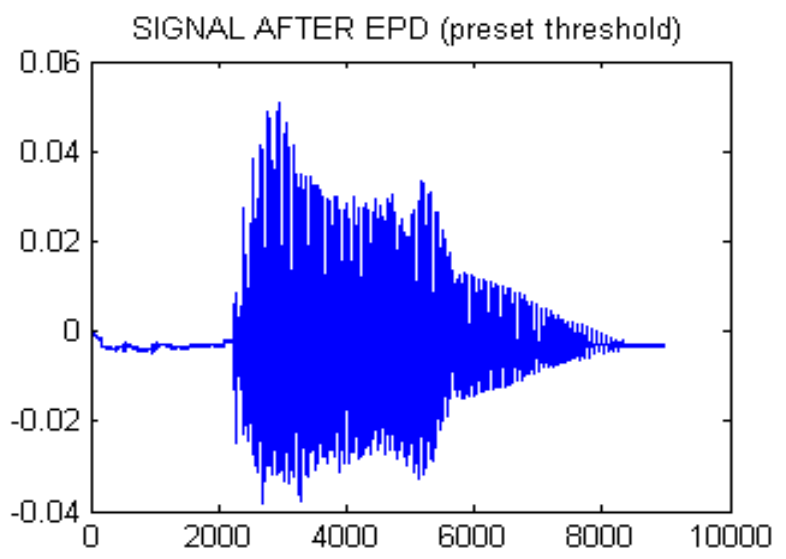

Figure 3: Waveform of isolated word (TEEN) by female speaker after endpoint detection (preset Threshold). 


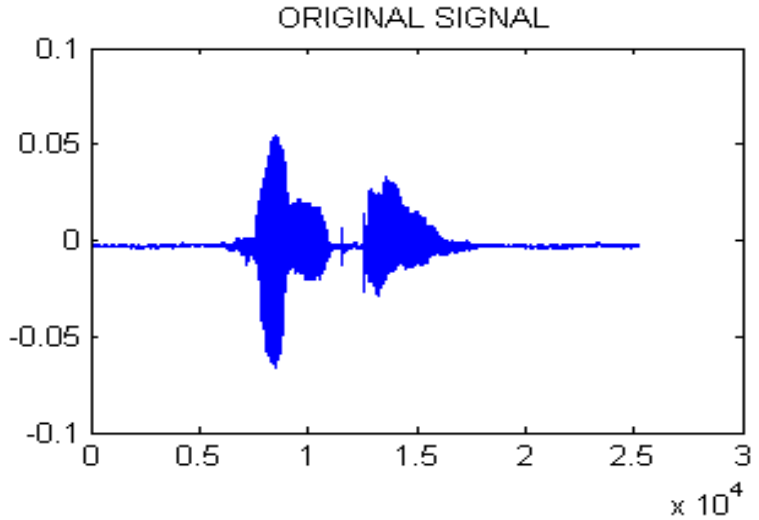

Figure 4: Waveform of paired word (HUM-TUM) by female speaker in original form.

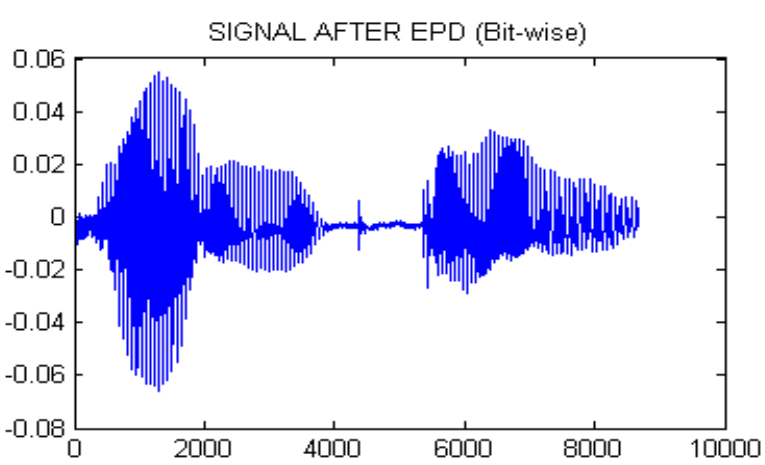

Figure 5: Waveform of paired word (HUM-TUM) by female speaker after endpoint detection (Bitwise).

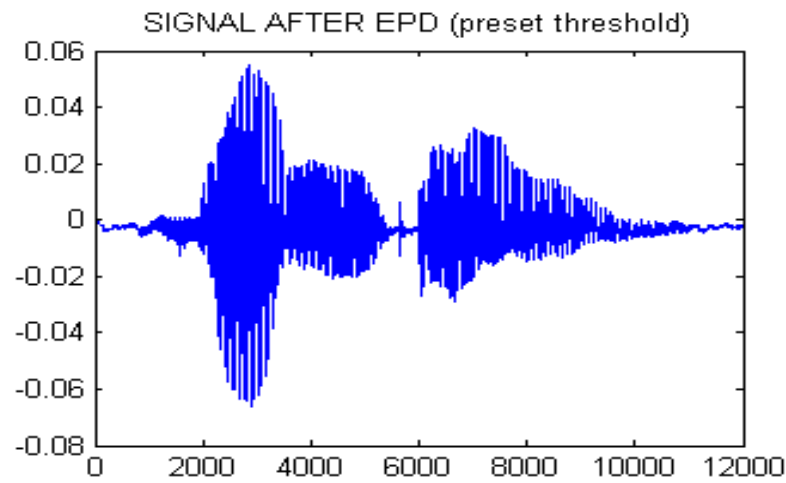

Figure 6: Waveform of paired word (HUM-TUM) by female speaker after endpoint detection (preset Threshold).

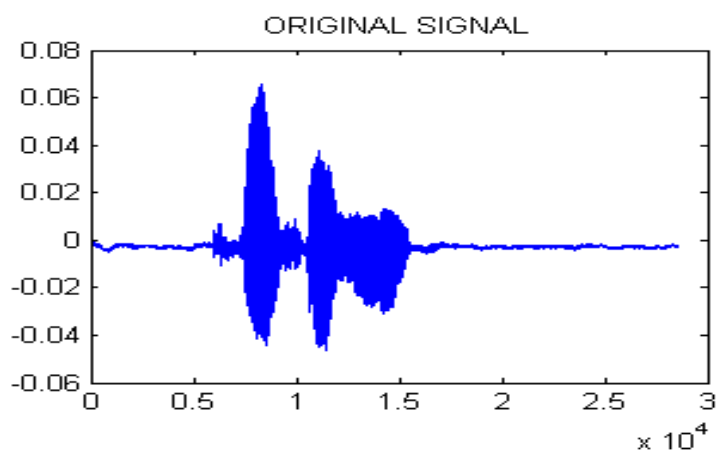

Figure 7: Waveform of Hybrid word (KHAS-AADMI) by female speaker in original form.

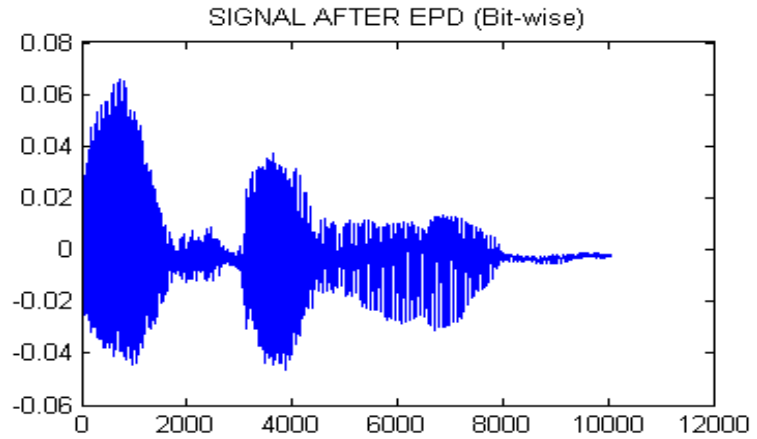

Figure 8: Waveform of Hybrid word (KHAS-AADMI) by female speaker after endpoint detection (Bitwise).

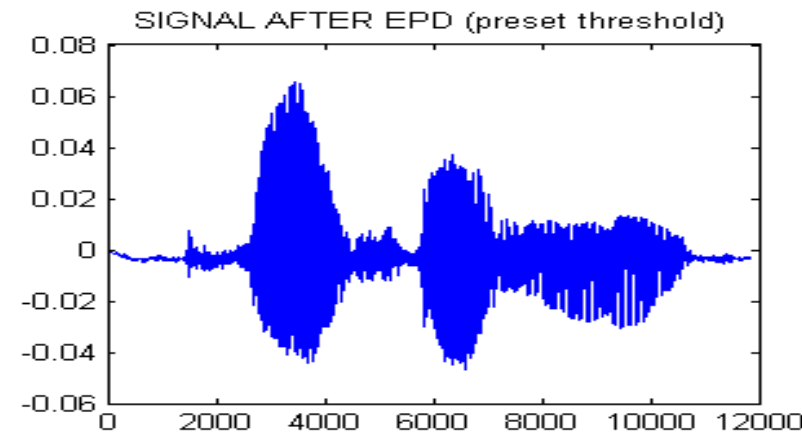

Figure9: Waveform of Hybrid word (KHASS-AADMI) by female Speaker after endpoint detection (preset Threshold).

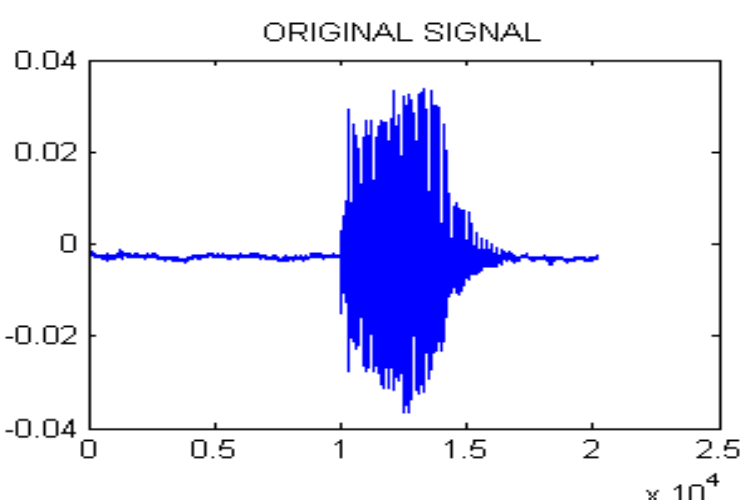

Figure 10: Waveform of isolated word (TEEN) by male speaker in original form.

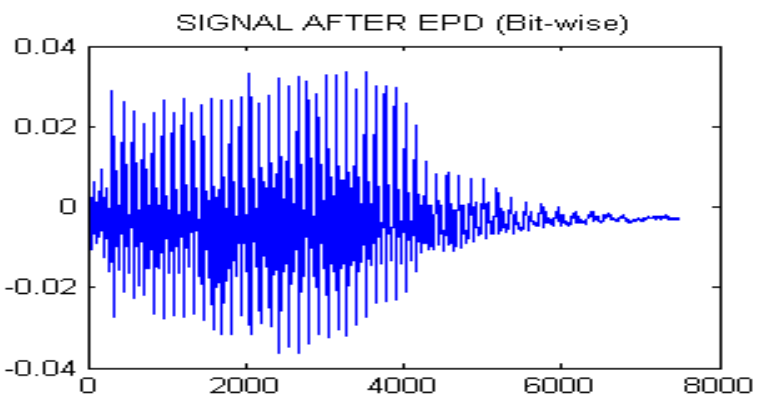

Figure- 11 Waveform of isolated word (TEEN) by male speaker after endpoint detection (Bit wise). 


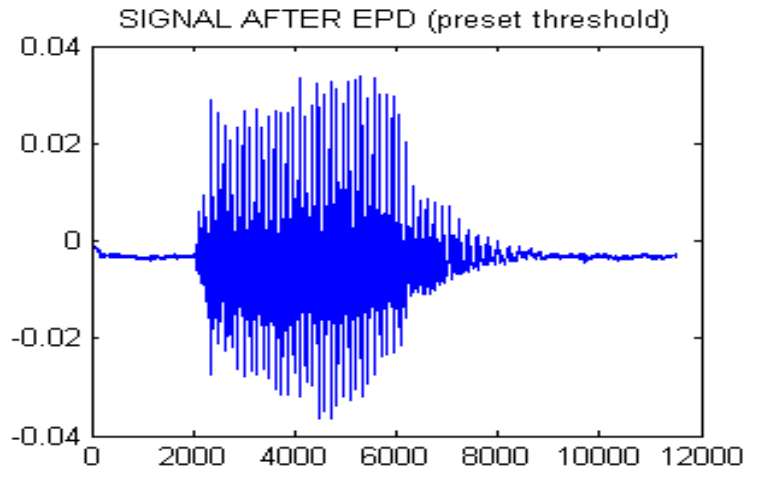

Figure 12: Waveform of isolated word (TEEN) by male speaker after endpoint detection (preset Threshold).

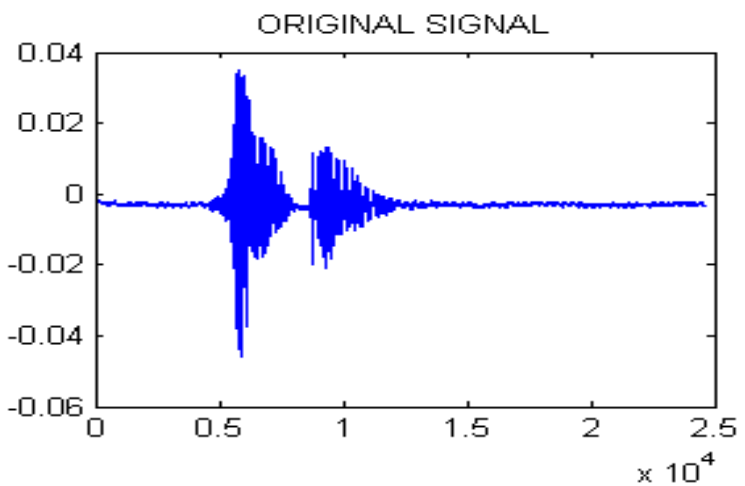

Figure 13: Waveform of paired word (HUM-TUM) by male Speaker in original form.

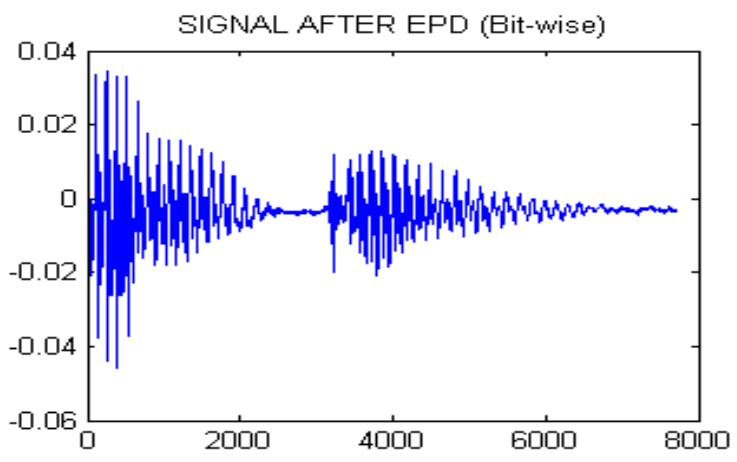

Figure 14: Waveform of paired word (HUM-TUM) by male speaker after endpoint detection (Bit wise).

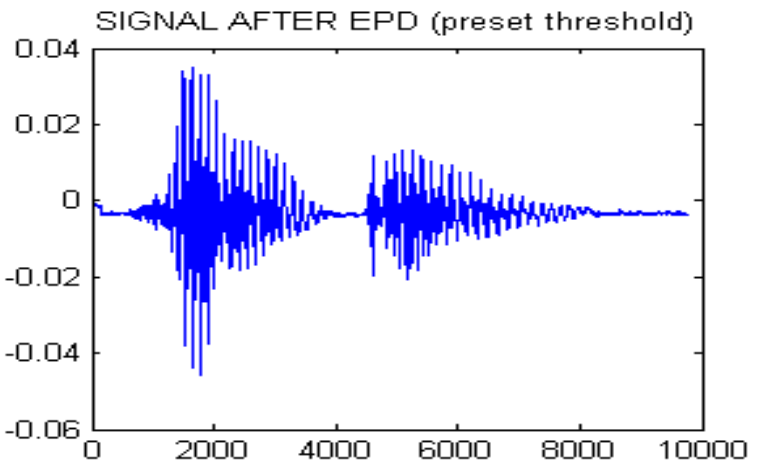

Figure 15: Waveform of paired word (HUM-TUM) by male speaker after endpoint detection (preset Threshold).

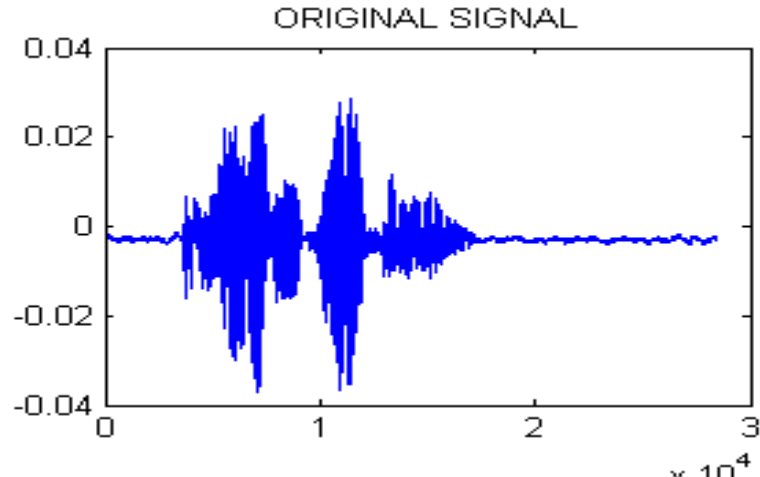

Figure 16: Waveform of Hybrid word (KHAS-AADMI) by male Speaker in original form.

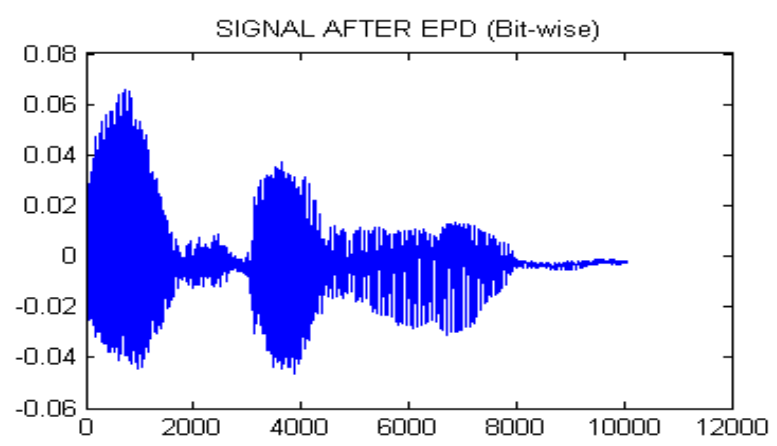

Figure 17: Waveform of Hybrid word (KHAS-AADMI) by male Speaker after endpoint detection (Bit wise).

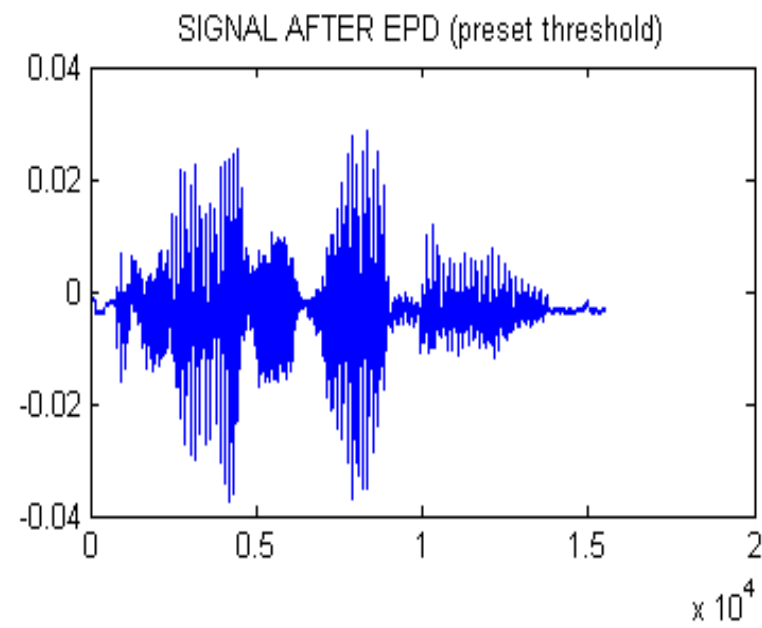

Figure 18: Waveform of Hybrid word (KHAS-AADMI) by male speaker after endpoint detection (preset Threshold). 
Table 1. Comparison of various parameters of female speakers for isolated words (average values).

\begin{tabular}{|c|c|c|c|c|c|c|c|c|}
\hline \multirow{2}{*}{ WORDS } & \multicolumn{7}{|c|}{ EPD USING PRESET THRESHOLD } & \multicolumn{3}{c|}{ EPD USING BIT BY BIT ANALYSIS } \\
& \multicolumn{7}{|c|}{ METHOD } & \multicolumn{2}{c|}{ TD } & RMS & MI \\
\cline { 2 - 9 } & NOS & TD & RMS & MI & NOS & & \\
\hline SHOONYA & 12416 & 0.776 & 0.016394 & 57.018 & 9054.8 & 0.565928 & 0.018936 & 58.25 \\
\hline EK & 11296 & 0.706 & 0.03593 & 58.742 & 7438 & 0.464878 & 0.051728 & 60.192 \\
\hline DO & 7968 & 0.498 & 0.01031 & 54.018 & 5455.8 & 0.34099 & 0.01207 & 55.368 \\
\hline TEEN & 9184 & 0.574 & 0.00869 & 52.532 & 6529 & 0.408064 & 0.009916 & 53.718 \\
\hline CHAR & 9824 & 0.614 & 0.00893 & 54.526 & 7931.2 & 0.495702 & 0.011942 & 55.346 \\
\hline PAANCH & 10272 & 0.642 & 0.015944 & 57.626 & 8004.6 & 0.520288 & 0.017846 & 58.58 \\
\hline CHEH & 9920 & 0.62 & 0.012438 & 55.56 & 8341.6 & 0.521386 & 0.013536 & 56.274 \\
\hline SAATH & 9408 & 0.588 & 0.01603 & 57.34 & 6264.8 & 0.391552 & 0.01949 & 58.954 \\
\hline AATH & 9120 & 0.57 & 0.014774 & 57.118 & 6329 & 0.395564 & 0.017426 & 58.568 \\
\hline NAU & 8320 & 0.52 & 0.011062 & 54.49 & 5767 & 0.360434 & 0.012452 & 55.524 \\
\hline
\end{tabular}

Table 2. Comparison of various parameters of female speakers for paired words (average values).

\begin{tabular}{|c|c|c|c|c|c|c|c|c|}
\hline \multirow[t]{2}{*}{ WORDS } & \multicolumn{4}{|c|}{$\begin{array}{c}\text { EPD USING PRESET THRESHOLD } \\
\text { METHOD }\end{array}$} & \multicolumn{4}{|c|}{ EPD USING BIT BY BIT ANALYSIS } \\
\hline & NOS & TD & RMS & MI & NOS & TD & RMS & MI \\
\hline ANGE- PINCHE & 14624 & 0.914 & 0.011136 & 54.32 & 13420.4 & 0.838768 & 0.011574 & 54.65 \\
\hline UPPER-NICHE & 14272 & 0.88 & 0.012386 & 55.05 & 12081.6 & 0.755102 & 0.0131378 & 55.59 \\
\hline DIN-RAAT & 13280 & 0.83 & 0.024152 & 58.57 & 10093.2 & 0.630824 & 0.028846 & 59.63 \\
\hline HUM-TUM & 11712 & 0.732 & 0.013649 & 56.24 & 9087 & 0.567936 & 0.0156 & 57.292 \\
\hline DHAN-DAULAT & 17728 & 1.108 & 0.014132 & 56.7 & 12559.4 & 0.784962 & 0.01669 & 57.986 \\
\hline JINA-MARNA & 15648 & 0.978 & 0.012938 & 55.564 & 12958 & 0.809876 & 0.013954 & 56.328 \\
\hline UTHANA-BAITHANA & 17216 & 1.076 & 0.0234118 & 59.482 & 12519 & 0.78244 & 0.027726 & 60.684 \\
\hline RANG-BERANG & 17216 & 1.076 & 0.014266 & 56.626 & 14617.7 & 0.913596 & 0.015584 & 57.286 \\
\hline KHANA-PINA & 16608 & 1.038 & 0.011266 & 54.48 & 15104.4 & 0.944024 & 0.011922 & 54.814 \\
\hline YANHA-WANHA & 15456 & 0.966 & 0.01326 & 55.432 & 11440.6 & 0.71504 & 0.014922 & 56.528 \\
\hline
\end{tabular}


Table 3. Comparison of various parameters of female speakers for Hybrid words (average values).

\begin{tabular}{|c|c|c|c|c|c|c|c|c|}
\hline \multirow[t]{2}{*}{ WORDS } & \multicolumn{4}{|c|}{$\begin{array}{c}\text { EPD USING PRESET THRESHOLD } \\
\text { METHOD }\end{array}$} & \multicolumn{4}{|c|}{ EPD USING BIT BY BIT ANALYSIS } \\
\hline & NOS & TD & RMS & MI & NOS & TD & RMS & MI \\
\hline KAALA-SAYA & 16896 & 1.056 & 0.033308 & 58.164 & 13526.4 & 0.8454 & 0.039328 & 59.016 \\
\hline KHAS-AADMI & 16768 & 1.048 & 0.021374 & 57.36 & 10828.6 & 0.676788 & 0.025694 & 58.996 \\
\hline BADIYA-ITEM & 16160 & 1.01 & 0.024438 & 58.412 & 12624.6 & 0.789039 & 0.027958 & 59.226 \\
\hline DOUBLE-ROTI & 13728 & 0.858 & 0.012306 & 55.02 & 12428.8 & 0.776682 & 0.012826 & 55.364 \\
\hline PURANA-ZAMANA & 16768 & 1.048 & 0.016336 & 57.114 & 13209.4 & 0.825592 & 0.017902 & 57.924 \\
\hline BADA-EHSAAN & 16325 & 1.022 & 0.021038 & 58.208 & 11892.4 & 0.743276 & 0.024334 & 59.328 \\
\hline BADIYA-JOKE & 14816 & 0.926 & 0.029444 & 58.176 & 11407 & 0.712938 & 0.032022 & 59.072 \\
\hline PURANI-JEANS & 18112 & 1.132 & 0.022064 & 57.468 & 14493.6 & 0.905364 & 0.024588 & 58.272 \\
\hline BADIYA-SAWAAL & 16704 & 1.044 & 0.016128 & 55.81 & 13876.4 & 0.867274 & 0.017196 & 56.324 \\
\hline TERI -IBADAT & 17056 & 1.066 & 0.017752 & 56.474 & 13583.2 & 0.848952 & 0.019832 & 57.348 \\
\hline
\end{tabular}

Table 4. Comparison of various parameters of male speakers for isolated words (average values).

\begin{tabular}{|c|c|c|c|c|c|c|c|c|}
\hline \multirow[t]{2}{*}{ WORDS } & \multicolumn{4}{|c|}{$\begin{array}{c}\text { EPD USING PRESET THRESHOLD } \\
\text { METHOD }\end{array}$} & \multicolumn{4}{|c|}{ EPD USING BIT BY BIT ANALYSIS } \\
\hline & NOS & TD & RMS & MI & NOS & TD & RMS & MI \\
\hline SHOONYA & 10484 & 0.664 & 0.012568 & 54.172 & 8717.4 & 0.544728 & 0.014323 & 54.892 \\
\hline EK & 8288 & 0.518 & 0.026034 & 59.48 & 6540.8 & 0.408802 & 0.026472 & 60.174 \\
\hline DO & 9280 & 0.58 & 0.065734 & 60.164 & 5892 & 0.368254 & 0.089972 & 61.784 \\
\hline TEEN & 9152 & 0.572 & 0.009406 & 53.136 & 6648.2 & 0.415512 & 0.010664 & 54.252 \\
\hline CHAR & 9120 & 0.57 & 0.012812 & 54.848 & 7666.6 & 0.479164 & 0.013988 & 55.426 \\
\hline PAANCH & 11936 & 0.746 & 0.045964 & 62.266 & 7995.8 & 0.499738 & 0.0592178 & 63.866 \\
\hline CHEH & 10720 & 0.67 & 0.045352 & 63.712 & 7268.4 & 0.454725 & 0.058856 & 65.154 \\
\hline SAATH & 10176 & 0.636 & 0.054674 & 63.762 & 7059.6 & 0.441224 & 0.064128 & 65.21 \\
\hline AATH & 10272 & 0.642 & 0.070688 & 61.198 & 7495 & 0.468438 & 0.082612 & 62.466 \\
\hline NAU & 9952 & 0.622 & 0.064746 & 60.828 & 5598.8 & 0.348828 & 0.10009 & 62.788 \\
\hline
\end{tabular}


Table 5. Comparison of various parameters of male speakers for paired words (average values).

\begin{tabular}{|c|c|c|c|c|c|c|c|c|}
\hline \multirow[t]{2}{*}{ WORDS } & \multicolumn{4}{|c|}{$\begin{array}{c}\text { EPD USING PRESET THRESHOLD } \\
\text { METHOD }\end{array}$} & \multicolumn{4}{|c|}{ EPD USING BIT BY BIT ANALYSIS } \\
\hline & NOS & TD & RMS & MI & NOS & TD & RMS & MI \\
\hline ANGE- PINCHE & 15008 & 0.938 & 0.010682 & 53.692 & 13066.6 & 0.816666 & 0.011284 & 54.136 \\
\hline UPPER-NICHE & 13824 & 0.864 & 0.013926 & 55.442 & 10278.8 & 0.642454 & 0.014712 & 55.988 \\
\hline DIN-RAAT & 13024 & 0.814 & 0.013106 & 54.938 & 9949.8 & 0.621864 & 0.01481 & 55.896 \\
\hline HUM-TUM & 9792 & 0.612 & 0.006716 & 50.256 & 8374.6 & 0.523414 & 0.007112 & 50.708 \\
\hline DHAN-DAULAT & 13728 & 0.858 & 0.010736 & 53.908 & 11509.2 & 0.719326 & 0.01145 & 54.572 \\
\hline JINA-MARNA & 15968 & 0.998 & 0.008186 & 51.625 & 11851 & 0.740688 & 0.009456 & 52.74 \\
\hline UTHANA-BAITHANA & 14016 & 0.874 & 0.010338 & 52.672 & 12563 & 0.78519 & 0.010608 & 52.904 \\
\hline RANG-BERANG & 13600 & 0.85 & 0.009114 & 52.076 & 10962.5 & 0.685328 & 0.009974 & 52.806 \\
\hline KHANA-PINA & 15520 & 0.97 & 0.008002 & 50.6 & 12713 & 0.794562 & 0.008592 & 51.166 \\
\hline YANHA-WANHA & 13760 & 0.86 & 0.00944 & 53.04 & 11078.6 & 0.692414 & 0.010394 & 53.808 \\
\hline
\end{tabular}

Table 6. Comparison of various parameters of male speakers for Hybrid words (average values).

\begin{tabular}{|c|c|c|c|c|c|c|c|c|}
\hline \multirow[t]{2}{*}{ WORDS } & \multicolumn{4}{|c|}{$\begin{array}{c}\text { EPD USING PRESET THRESHOLD } \\
\text { METHOD }\end{array}$} & \multicolumn{4}{|c|}{ EPD USING BIT BY BIT ANALYSIS } \\
\hline & NOS & TD & RMS & MI & NOS & TD & RMS & MI \\
\hline KAALA-SAYA & 15776 & 0.986 & 0.010098 & 53.098 & 12069.6 & 0.754352 & 0.011712 & 54.11 \\
\hline KHAS-AADMI & 15840 & 0.99 & 0.00885 & 51.576 & 12889.6 & 0.80574 & 0.009778 & 52.24 \\
\hline BADIYA-ITEM & 14048 & 0.878 & 0.01022 & 53.482 & 11788 & 0.7367516 & 0.0112762 & 54.16 \\
\hline DOUBLE-ROTI & 15040 & 0.94 & 0.00691 & 50.422 & 11770.4 & 0.735652 & 0.00766 & 51.226 \\
\hline PURANA-ZAMANA & 16928 & 1.058 & 0.007182 & 50.974 & 14181.2 & 0.886328 & 0.007662 & 51.54 \\
\hline BADA-EHSAAN & 15974 & 1.004 & 0.008236 & 51.798 & 12619.8 & 0.788736 & 0.009038 & 52.576 \\
\hline BADIYA-JOKE & 13504 & 0.844 & 0.0104 & 53.406 & 10469.8 & 0.654364 & 0.011834 & 54.318 \\
\hline PURANI-JEANS & 16704 & 1.044 & 0.00648 & 49.804 & 14095.8 & 0.880988 & 0.006864 & 50.242 \\
\hline BADIYA-SAWAAL & 14016 & 0.876 & 0.006332 & 49.804 & 12139.6 & 0.758926 & 0.006598 & 50.162 \\
\hline TERI -IBADAT & 15136 & 0.946 & 0.008112 & 51.798 & 13577.2 & 0.848576 & 0.008514 & 52.176 \\
\hline
\end{tabular}


Table 7. Increment $(\uparrow)$ and Decrement $(\downarrow)$ of all parameters in case of female speakers for Isolated words (in percentage).

\begin{tabular}{|c|c|c|c|c|}
\hline WORDS & NOS & TD & RMS & MI \\
\hline SHOONYA & $27.0712(\downarrow)$ & $27.07113(\downarrow)$ & $15.50567(\uparrow)$ & $2.16072(\uparrow)$ \\
\hline EK & $34.15368(\downarrow)$ & $34.15326(\downarrow)$ & $43.96883(\uparrow)$ & $2.46842(\uparrow)$ \\
\hline DO & $31.52861(\downarrow)$ & $31.52811(\downarrow)$ & $17.07081(\uparrow)$ & $2.49917(\uparrow)$ \\
\hline TEEN & $28.90987(\downarrow)$ & $28.90871(\downarrow)$ & $14.10817(\uparrow)$ & $1.50387(\uparrow)$ \\
\hline CHAR & $19.26710(\downarrow)$ & $19.26645(\downarrow)$ & $33.72900(\uparrow)$ & $1.65550(\uparrow)$ \\
\hline PAANCH & $22.07359(\downarrow)$ & $22.07352(\downarrow)$ & $8.82779(\uparrow)$ & $1.28509(\uparrow)$ \\
\hline CHEH & $15.91129(\downarrow)$ & $15.90548(\downarrow)$ & $21.58453(\uparrow)$ & $2.81479(\uparrow)$ \\
\hline SAATH & $33.40986(\downarrow)$ & $33.40982(\downarrow)$ & $17.95045(\uparrow)$ & $2.52860(\uparrow)$ \\
\hline AATH & $30.60307(\downarrow)$ & $30.60281(\downarrow)$ & $12.56554(\uparrow)$ & $1.89759(\uparrow)$ \\
\hline NAU & $30.68509(\downarrow)$ & $30.68577(\downarrow)$ & & \\
\hline
\end{tabular}

Table 8. Increment $(\uparrow)$ and Decrement $(\downarrow)$ of all parameters in case of female speakers for Paired words (in percentage).

\begin{tabular}{|c|c|c|c|c|}
\hline WORDS & NOS & TD & RMS & MI \\
\hline ANGE- PINCHE & $8.23031(\downarrow)$ & $8.23107(\downarrow)$ & $3.93319(\uparrow)$ & $0.60751(\uparrow)$ \\
\hline UPPER-NICHE & $15.34753(\downarrow)$ & $14.19295(\downarrow)$ & $6.06976(\uparrow)$ & $0.98093(\uparrow)$ \\
\hline DIN-RAAT & $23.99699(\downarrow)$ & $23.99711(\downarrow)$ & $19.43524(\uparrow)$ & $1.80980(\uparrow)$ \\
\hline HUM-TUM & $22.41291(\downarrow)$ & $22.41311(\downarrow)$ & $13.91850(\uparrow)$ & $1.87055(\uparrow)$ \\
\hline DHAN-DAULAT & $29.15501(\downarrow)$ & $29.15505(\downarrow)$ & $18.10076(\uparrow)$ & $2.26807(\uparrow)$ \\
\hline JINA-MARNA & $17.19069(\downarrow)$ & $17.01959(\downarrow)$ & $7.85284(\uparrow)$ & $1.37499(\uparrow)$ \\
\hline UTHANA-BAITHANA & $27.28276(\downarrow)$ & $27.28253(\downarrow)$ & $18.42746(\uparrow)$ & $2.02078(\uparrow)$ \\
\hline RANG-BERANG & 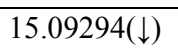 & $15.09330(\downarrow)$ & $9.23875(\uparrow)$ & $1.16554(\uparrow)$ \\
\hline KHANA-PINA & $9.05347(\downarrow)$ & $9.05356(\downarrow)$ & $5.82283(\uparrow)$ & $0.61307(\uparrow)$ \\
\hline YANHA-WANHA & $25.97955(\downarrow)$ & $25.97929(\downarrow)$ & $12.53394(\uparrow)$ & $1.97719(\uparrow)$ \\
\hline
\end{tabular}


Table 9. Increment $(\uparrow)$ and Decrement $(\downarrow)$ of all parameters in case of female speakers for Hybrid words (in percentage).

\begin{tabular}{|c|c|c|c|c|}
\hline WORDS & NOS & TD & RMS & MI \\
\hline KAALA-SAYA & $19.94318(\downarrow)$ & $19.94318(\downarrow)$ & $18.07374(\uparrow)$ & $1.46482(\uparrow)$ \\
\hline KHAS-AADMI & $35.42104(\downarrow)$ & $35.42099(\downarrow)$ & $20.21147(\uparrow)$ & $2.85216(\uparrow)$ \\
\hline BADIYA-ITEM & $21.87747(\downarrow)$ & $21.87733(\downarrow)$ & $14.40379(\uparrow)$ & $1.39355(\uparrow)$ \\
\hline DOUBLE-ROTI & $9.46387(\downarrow)$ & $9.47762(\downarrow)$ & $4.22588(\uparrow)$ & $0.62523(\uparrow)$ \\
\hline PURANA-ZAMANA & $21.22257(\downarrow)$ & $21.22214(\downarrow)$ & $9.58619(\uparrow)$ & $1.41822(\uparrow)$ \\
\hline BADA-EHSAAN & $27.27250(\downarrow)$ & $27.27241(\downarrow)$ & $15.66689(\uparrow)$ & $1.92413(\uparrow)$ \\
\hline BADIYA-JOKE & $23.00891(\downarrow)$ & $23.00886(\downarrow)$ & $8.75560(\uparrow)$ & $1.54015(\uparrow)$ \\
\hline PURANI-JEANS & $19.97792(\downarrow)$ & $20.02085(\downarrow)$ & $11.43945(\uparrow)$ & $1.39904(\uparrow)$ \\
\hline BADIYA-SAWAAL & $16.92768(\downarrow)$ & $16.92778(\downarrow)$ & $6.62202(\uparrow)$ & $0.95323(\uparrow)$ \\
\hline TERI -IBADAT & $20.36116(\downarrow)$ & $20.36098(\downarrow)$ & $11.71699(\uparrow)$ & $1.54761(\uparrow)$ \\
\hline
\end{tabular}

Table 10. Increment $(\uparrow)$ and Decrement $(\downarrow)$ of all parameters in case of male speakers for Isolated words (in percentage).

\begin{tabular}{|c|c|c|c|c|}
\hline WORDS & NOS & TD & RMS & MI \\
\hline SHOONYA & $16.87905(\downarrow)$ & $17.96265(\downarrow)$ & $13.96404(\uparrow)$ & $1.32909(\uparrow)$ \\
\hline EK & $21.08108(\downarrow)$ & $21.08069(\downarrow)$ & $1.69009(\uparrow)$ & $2.27234(\uparrow)$ \\
\hline DO & $36.50862(\downarrow)$ & $36.50793(\downarrow)$ & $36.87285(\uparrow)$ & $2.69264(\uparrow)$ \\
\hline TEEN & $27.35794(\downarrow)$ & $27.35804(\downarrow)$ & $13.37444(\uparrow)$ & $1.05382(\uparrow)$ \\
\hline CHAR & $15.93640(\downarrow)$ & $15.93641(\downarrow)$ & $9.17889(\uparrow)$ & $2.56962(\uparrow)$ \\
\hline PAANCH & $33.01106(\downarrow)$ & $33.01099(\downarrow)$ & $28.83561(\uparrow)$ & $2.26331(\uparrow)$ \\
\hline CHEH & $32.19776(\downarrow)$ & $32.13059(\downarrow)$ & $29.77578(\uparrow)$ & $2.27095(\uparrow)$ \\
\hline SAATH & $30.625(\downarrow)$ & $30.62516(\downarrow)$ & $17.29158(\uparrow)$ & $2.07196(\uparrow)$ \\
\hline AATH & $27.03466(\downarrow)$ & $27.03458(\downarrow)$ & $16.86849(\uparrow)$ & $3.22220(\uparrow)$ \\
\hline NAU & $43.74196(\downarrow)$ & $43.74148(\downarrow)$ & $54.5887(\uparrow)$ & \\
\hline
\end{tabular}


Table 11. Increment $(\uparrow)$ and Decrement $(\downarrow)$ of all parameters in case of male speakers for Paired words (in percentage).

\begin{tabular}{|c|c|c|c|c|}
\hline WORDS & NOS & TD & RMS & MI \\
\hline ANGE- PINCHE & $12.93574(\downarrow)$ & $12.93539(\downarrow)$ & $5.635652(\uparrow)$ & $0.82694(\uparrow)$ \\
\hline UPPER-NICHE & $25.64525(\downarrow)$ & $25.64189(\downarrow)$ & $5.64412(\uparrow)$ & $0.98481(\uparrow)$ \\
\hline DIN-RAAT & $23.60412(\downarrow)$ & $23.60393(\downarrow)$ & $13.00168(\uparrow)$ & $1.74378(\uparrow)$ \\
\hline HUM-TUM & $14.47508(\downarrow)$ & $14.47484(\downarrow)$ & $5.89637(\uparrow)$ & $0.89939(\uparrow)$ \\
\hline DHAN-DAULAT & $16.16259(\downarrow)$ & $16.16247(\downarrow)$ & $6.65052(\uparrow)$ & $1.23173(\uparrow)$ \\
\hline JINA-MARNA & $25.78282(\downarrow)$ & $25.78277(\downarrow)$ & $15.51429(\uparrow)$ & $2.10640(\uparrow)$ \\
\hline UTHANA-BAITHANA & $11.08019(\downarrow)$ & $10.16133(\downarrow)$ & $2.61172(\uparrow)$ & $0.44046(\uparrow)$ \\
\hline RANG-BERANG & $19.39338(\downarrow)$ & $19.37318(\downarrow)$ & $9.43603(\uparrow)$ & $1.40179(\uparrow)$ \\
\hline KHANA-PINA & $18.08634(\downarrow)$ & $18.08639(\downarrow)$ & $7.37316(\uparrow)$ & $2.09486(\uparrow)$ \\
\hline YANHA-WANHA & $19.48692(\downarrow)$ & $19.48674(\downarrow)$ & $10.10593(\uparrow)$ & $1.44796(\uparrow)$ \\
\hline
\end{tabular}

Table 12. Increment $(\uparrow)$ and Decrement $(\downarrow)$ of all parameters in case of male speakers for Hybrid words (in percentage).

\begin{tabular}{|c|c|c|c|c|}
\hline WORDS & NOS & TD & RMS & MI \\
\hline KAALA-SAYA & $23.49391(\downarrow)$ & $23.49371(\downarrow)$ & $15.98336(\uparrow)$ & $1.90591(\uparrow)$ \\
\hline KHAS-AADMI & $18.62626(\downarrow)$ & $18.61212(\downarrow)$ & $10.48588(\uparrow)$ & $1.28742(\uparrow)$ \\
\hline BADIYA-ITEM & $16.08769(\downarrow)$ & $16.08762(\downarrow)$ & $10.33464(\uparrow)$ & $1.26772(\uparrow)$ \\
\hline DOUBLE-ROTI & $21.73936(\downarrow)$ & $21.73915(\downarrow)$ & $10.85384(\uparrow)$ & $1.59454(\uparrow)$ \\
\hline PURANA-ZAMANA & $16.22637(\downarrow)$ & $16.22609(\downarrow)$ & $6.68338(\uparrow)$ & $1.11037(\uparrow)$ \\
\hline BADA-EHSAAN & $20.99787(\downarrow)$ & $21.44064(\downarrow)$ & $9.73774(\uparrow)$ & $1.50199(\uparrow)$ \\
\hline BADIYA-JOKE & $22.46889(\downarrow)$ & $22.46872(\downarrow)$ & $13.78846(\uparrow)$ & $1.70767(\uparrow)$ \\
\hline PURANI-JEANS & $15.61422(\downarrow)$ & $15.61418(\downarrow)$ & $5.92593(\uparrow)$ & $0.87945(\uparrow)$ \\
\hline BADIYA-SAWAAL & $13.38356(\downarrow)$ & $13.36461(\downarrow)$ & $4.20088(\uparrow)$ & $0.71882(\uparrow)$ \\
\hline TERI -IBADAT & $10.29863(\downarrow)$ & $10.29852(\downarrow)$ & $4.95562(\uparrow)$ & $0.71238(\uparrow)$ \\
\hline
\end{tabular}

\section{CONCLUSION}

A new Bit wise approach for detecting the accurate endpoints of a speech signal is presented. Complete details regarding the implementation of the algorithm have been provided. The experimental results demonstrate that the proposed algorithm can be used to enhance the performance of endpoint detection algorithms. The result shows average reduction of $27.36 \%$ and $27.36 \%$ for Isolated words, $19.37 \%$ and $19.26 \%$ for Paired words and $21.55 \%$ and $21.55 \%$ for Hybrid words in NOS and TD, while average enhancement of $19.72 \%$ and $2.11 \%$ for Isolated words, $11.53 \%$ and $1.47 \%$ for Paired words and
$12.07 \%$ and $1.51 \%$ for Hybrid words in RMS and MI values for female speakers respectively. For male speakers an average reduction of $28.44 \%$ and $28.54 \%$ for Isolated words, $18.67 \%$ and $18.57 \%$ for Paired words and $17.89 \%$ and $17.93 \%$ for Hybrid words in NOS and TD, while as an average enhancement of $22.24 \%$ and $2.08 \%$ for Isolated words, $8.19 \%$ and $1.32 \%$ for Paired words and $9.29 \%$ and $1.27 \%$ for Hybrid words in RMS and MI is seen respectively. Due to reduction in NOS and TD, processing time will be reduced and enhancement in RMS and MI values increases 
the information content. The proposed Bit-wise endpoint detection method may lead to higher recognition rates.

\section{REFERENCES}

[1] Biing,H.J. and Sadaoki,F. 2000, Automatic recognition and understanding of Spoken launguage- A first step towards natural human-machine communication, Proceedings of the IEEE Vol.88.

[2] Rajoriya, D.K., Anand, R.S. and Maheshwari R.P. 2010, Hindi paired word recognition using probabilistic neural network, International Journal Computational Intelligence studies, Vol.1.

[3] Cowie, R. and Cornelius, R.R. 2003, Describing the emotional states that are expressed in speech, Speech Communication, Elsevier, Vol. 40.

[4] Rajoriya, D.K., Anand, R.S. and Maheshwari, R.P. 2011,Spoken Paired Word Pattern Classification Using Whole Word Template, TECHNIA- International Journal of Computing Science and Communication Technologies, Vol.3.

[5] Saon, G. and Padmanabham, M. 2001,Data-driven approach to designing compound words for continuous speech recognition, IEEE Transactions on Speech and Audio Processing,Vol. 9,No.4, pp.327-332.

[6] Sasaki, S. and Matsumoto, I. 1994, Voice activity detection and transmission error control for digital cordless telephone system, IEICE Trans.Commun., Vol. E77B, no. 7,1994; pp. 948-955.

[7] Hariharan, R., Hakkinan, J. and Laurila, K.200), Robust end of utterance detection for real time speech recognition applications, IEEE International conference on Acoustics, Speech and Signal Processing.

[8] Singh, A., Rajoriya, D.K. and Singh, V. 2012, Database Development and Analysis of Spoken Hindi Hybrid Words Using Endpoint Detection, International Journal $j$ of Electronics and Computer Science Engineering, Vol.1.

[9] Bullington, K. and Fraser, J.M. 1959, Engineering aspects of TASI, Bell Syst.Tech. J'., pp. 353-364.

[10] Ying, G.S.,Mitchell, C.D.,Jamieson, L.H. 1992, Endpoint Detection of Isolated Utterances Based on A Modified Teager Energy Measurement. In Proc. IEEE ICASSP-92, pp.732-pp.735.
[11] Atal, B.and Rabiner, L.R. 1976,A pattern recognition approach to voiced-unvoiced-silence classification with applications to speech recognition, Acoustics, Speech, and Signal Processing [see also IEEE Transactions on Signal Processing], IEEE Transactions on, Volume: 24 , Issue: 3 , Pages: $201-212$

[12] Childers, D.G.,Hand, M. and Larar J.M. 1989, Silent and Voiced/Unvoied/ Mixed Excitation(Four-Way), Classification of Speech, IEEE Transaction on ASSP, Vol-37, No-11, pp. 1771-74.

[13] Halverson, D.R. 1995 , Robust estimation and signal detection with dependent nonstationary data, Circuits Syst. Signal Process., vol. 14, no.4,pp.465-472.

[14] Rabiner, L.R. and Juang, B.H 1993, Fundamentals of speech recognition, 1st Indian Reprint, Pearson Education.

[15] Wilpon, J.G.,Rabiner, L.R. and Martin, T. 1984, An improved word-detection algorithm for telephone-quality speech incorporating both syntactic and semantic constraints, AT\&T Bell Labs. Tech. J., vol. 63, pp. 479498.

[16] Chengalvarayan, R. 1999, Robust energy normalization using speech/non speech discriminator for German connected digit recognition, in Proc.Eurospeech'99, Budapest, Hungary, pp. 61-64.

[17] Rabiner, L.R. and Sambur, M.R. 1975, An algorithm for determining the endpoints of isolated utterance's, Bell Syst. Tech. J., vol. 54, pp. 297-315.

[18] Junqua, J.C.,Reaves, B. And Mak B. 1991, A study of endpoint detection algorithms in adverse conditions: Incidence on a DTW and HMM recognize ,in Proc. Eurospeech, pp. 1371-1374

[19] Qi Li, Zheng, J., Tsai, A.and Zhou, Q. 2002, Robust Endpoint Detection and Energy Normalization for RealTime Speech and Speaker Recognition, IEEE Transactions on speech and audio processing, Vol.10, NO.3.

[20] Tanyer, S.G. and Özer, H. 2000, Voice Activity Detection in Non Stationary Noise, IEEE Transactions on speech and audio processing, Vol. 8, NO. 4.

[21] Qi.Li and Tsai, A. 1999, A language- independent personal voice controller with embedded speaker verification, in Proc. Eurospeech'99, Budapest, Hungary. 